\title{
Molecular characterization of Fusarium oxysporum f. sp. cubense Tropical Race 4 (Foc- TR4) isolates from Malaysian banana using secreted in Xylem (SIX) effector genes
}

\begin{abstract}
Fusarium wilt of banana, caused by Fusarium oxysporum f. sp. cubense (Foc), is a major factor limiting banana production in Malaysia. To date, SSR, ISSR, AFLP and RAPD markers are widely used in PCR-based molecular characterization of Foc. Although these markers are effective in characterizing Foc, they all have their own limitations. An effector gene known as Secreted in Xylem (SIX) has been explored to broaden the molecular diagnostic toolbox for Foc. This study aims to investigate the diversity of Foc-TR4 in Peninsular Malaysia based on the phylogenetic analysis of SIX sequences. The virulence potential of twenty-seven Fusarium wilt of banana based on pathogenicity test and the presence of SIX1, SIX7 and SIX8 genes were determined. All of twenty-seven Foc 'Tropical Race 4' (TR4) (VCG 01213/160) isolates were obtained from Biological Control Laboratory, Department of Plant Protection, Universiti Putra Malaysia (UPM). The results showed that all Foc-TR4 isolates were pathogenic towards banana plantlets at different severity levels under greenhouse condition. Pathogenicity assays showed that the level of aggressiveness differs between isolates. The Foc-TR4 isolates were screened for the presence of three SIX genes (SIX1, SIX7 and SIX8) and the genetic differentiation of Foc-TR4 was evaluated by PCR analysis using three specific primers. Among these three SIX genes, SIX1 and SIX8 genes were detected in all twenty-seven Foc-TR4 isolates from Peninsular Malaysia. Phylogenetic analysis using SIX1 and SIX8 sequences showed that they were related to TR4 isolates (VCG 01213/16) from Australia and Indonesian with bootstrap values of $94 \%$ and $100 \%$ respectively. No variation was observed in the SIX1 and SIX8 sequences since all 27 isolates were clustered into the same clade. Virulence level also did not correlate with the existence or lack of these genes. Since Foc could hamper the local banana production in Malaysia, characterization of these isolates through SIX genes provides a great understanding of pathogenicity in Foc and could potentially improve the diagnostic and control of Fusarium wilt in the future.
\end{abstract}

Keyword: Banana; Fusarium oxysporum f. sp. Cubense; Fusarium wilt; Secreted in Xylem (SIX) 
